\title{
Personality and Intrinsic Motivational Factors in End-User Programming
}

\author{
Saeed Aghaee, Alan F. Blackwell, David Stillwell \\ University of Cambridge \\ Cambridge, UK
}

\author{
Michal Kosinski \\ Stanford Graduate School of Business \\ Stanford, CA, USA
}

\begin{abstract}
We explore the factors that determine whether individuals are likely to experience intrinsic motivation in enduser programming (EUP). We report two experiments: one that tests whether there are reliable psychometric constructs that describe different aspects of intrinsic motivation, and one that tests whether these constructs are successful in predicting individuals' own self-reported intrinsic motivation after using a popular EUP product. We conclude that there are identifiable and distinct motivational factors in EUP, and that these are associated with particular psychometric personality traits. We offer several suggestions for future research that could apply these findings to improve uptake and quality of user experience for educational and general-purpose EUP applications.
\end{abstract}

Keywords-end-user programming, motivation, personality

\section{INTRODUCTION}

A key objective in End-User Programming (EUP) research, as in the VL/HCC conference series more broadly, is to provide people with the capability to create and modify software. In particular, the goal of EUP is to extend that capability to a wider range of people, beyond professional programmers, in a way that will help these people achieve useful things in the world. This objective was expressed in the title of the EUSES research consortium: End-Users Shaping Effective Software. However, as was often noted in presentations by the EUSES consortium director Margaret Burnett, a key question arises whether "If we build it, will they come?" [1].

This paper describes a research programme that sets out to evaluate that specific question. There has been some prior research, at $\mathrm{VL} / \mathrm{HCC}$ and related venues, exploring questions such as self-efficacy (the belief that one will be able to accomplish a task). We review that research briefly in the next section. However, in this project, we have chosen to explore the question from a new perspective, applying the methods of psychometric personality testing. In this case, we are not asking more generally about the personality of programmers (although this has been done before). Rather, we are asking what measurable aspect of end-user personality might help us to answer the question "will they come?" to EUP. This use of personality to understand motivation reflects a shift in the intrinsic motivation literature (e.g. at the Nebraska Symposium on Motivation) from understanding what things are motivating, to investigating who is motivated by them.
In more precise terms, our research questions are as follows:

RQ1: What are the personality factors that might cause EUP to be intrinsically motivating for some group of individuals?

RQ2: How can we characterize and measure, for any individual, the particular factors that will cause EUP to be intrinsically motivating for that person?

The approach that we take is a psychometric one - that is to say, we are interested in psychological factors that can be numerically measured, and hence used for comparison and prediction of individual preferences and behavior.

The outline of the rest of the paper is as follows. We provide a brief literature review of previous research related to intrinsic motivation in EUP, and research background to the psychometric techniques that we use. We then propose a number of hypothetical factors that might be involved in intrinsic motivation, as expected from prior literature. In a first study, exploring RQ1, we validated these factors against a large dataset of personality scores collected from Facebook users. In a second study, exploring RQ2, new users of a popular end-user programming tool were asked to reflect on their experiences of using it, in order to evaluate whether those experiences were consistent with predictions derived from our model of intrinsic motivation factors.

\section{PRevious Research}

Although there has been much speculation about the personality of professional programmers, this may be of limited relevance to the factors that are intrinsically-motivating for end-user programmers. For example, some evidence indicates that programming aptitude correlates with diagnosis of developmental disorders such as those on the autism spectrum [4]. In the career planning context, the Myers-Briggs Type Indicator (MBTI) is sometimes used to assess suitability for professional work, and this has been done both for programming [5] and software engineering [6].

\section{A. Intrinsic Motivation in End-User Programming}

In the case of EUP, we are particularly interested in those who are not professional programmers, but might still engage in some kinds of programming activity. In this case, a key consideration is self-efficacy - the belief whether one is able to 
succeed in an activity that one has not previously attempted. Previous research has found that self-efficacy in EUP tasks is correlated with gender [7], thus contributing to exclusion of females from career and educational opportunities. In this research, we address psychometric variables that are also likely to be correlated with gender, and contribute to exclusion. However, we will not focus on the question of gender in the present study, largely because we want to avoid any temptation to interpret our findings through biological determinism.

Self-efficacy is also significant for EUP more broadly, because low self-efficacy modifies the perceived cost and risks in attention investment decisions [8], with the result that individuals with low self-efficacy are unlikely to commence a programming activity that could be beneficial to them. Attention investment analysis offers an account of EUP with regard to extrinsic motivation - what benefit does the end-user perceive from programming? In contrast, we are concerned with intrinsic motivation, which has received little attention so far, and more likely to be determined by personality factors, rather than external benefits.

\section{B. Psychometric Approach}

In order to study these personality factors, we adopt the widely used Five Factor Model of personality (FFM), encompassing five broad traits representing a basic, stable, and heritable structure underlying variations in human behavior and preferences. FFM has been shown to subsume most of the personality traits that had been identified in earlier research [2], [3]. The Five Factor Model comprises the following dimensions:

Openness to experience $(\mathrm{O})$ : Openness relates to imagination, creativity, curiosity, tolerance, political liberalism, and appreciation for culture. People scoring high on Openness like change, appreciate new and unusual ideas, and have a good sense of aesthetics. People low on Openness tend to be conservative, traditional, and disinterested in abstract ideas.

Conscientiousness (C): Conscientiousness measures the preference for an organized approach to life in contrast to a spontaneous one. Conscientious people are more likely to be reliable, consistent, enjoy planning, seek achievements, and pursue long-term goals. Non-conscientious individuals are generally more easy-going, spontaneous, and creative. They tend to be more tolerant and less bound by rules and plans.

Extroversion (E): Extroverts tend to seek stimulation in the external world, the company of others, and tend to express positive emotions. They are more energetic, talkative, outgoing, do not mind being the center of attention, and make new friends easily. Introverts tend to be solitary or reserved, and seek environments characterized by lower levels of external stimulation.

Agreeableness (A): Agreeableness relates to a focus on maintaining positive social relations, being compassionate, and cooperative. Agreeable people tend to be trustful and adapt to others' needs. Disagreeable people are more assertive, uncompromising, and focused on themselves. They also tend to be less gullible and are less bound by social expectations and conventions.
Emotional Stability: This trait, reversely referred to as "Neuroticism" (N), measures the tendency to experience mood swings and emotions, such as guilt, anger, anxiety, and depression. Emotionally stable people tend to be calmer and self-confident, whereas neurotic individuals are more likely to experience stress and nervousness.

\section{THEORY AND HYPOTHESES}

In this section, we develop a hypothetical model of intrinsic motivation in EUP, suitable for expression in terms of FFM personality factors, and thus able to be evaluated using psychometric techniques.

Our objective was to construct several alternative accounts, describing different types of intrinsic motivation that people might have for EUP. Each of these accounts should be defined in a way that clearly differentiates it from the others, and should offer plausible justifications in relation to popular culture (which we assume reflects some kinds of intrinsic motivation) and in terms of prior research literature in HCI or end-user programming.

We did not assume that the resulting set of intrinsic motivation types would be definitive, or exhaustive. The overall logic of the study is that these descriptions should serve as hypothetical component vectors within the multidimensional space of personality factors that underlie intrinsic motivation. We can use psychometric methods to test these components for independence. If we find evidence that the vectors are independent, then this supports the distinctions that we have drawn. However, as with all methods for dimensional reduction in statistics, it should be remembered that there may be alternative sets of orthogonal axes that describe the underlying space equally well.

We constructed these hypothetical motivation factors based on prior research in EUP and HCI, together with popular culture accounts of programming. The objective was descriptive, with the goal that each factor should be associated with a distinctive label - a descriptive term that could reliably be used in analysis without conceptual drift between definition and interpretation of results. The process was iterative, with each iteration involving: a) collect phenomena to be described; b) group by similarity; c) assign provisional labels; d) merge or divide groups as required to clarify labels definitions; e) test by collection and assignment of new phenomena. The final outcome of this qualitative analytic process was the following set of three hypothetical personality factors:

\section{A. Bricoleurism}

The term 'bricoleur' has several implications for end-user programming. One is the writing of Claude Levi-Strauss, which makes a distinction between the (formally educated) engineer and the (improvising, informal) bricoleur. Another is the contemporary French use of the word 'bricolage' corresponding to the modern English acronym DIY. There is an allusion to both of these in writing by Turkle and Papert [9] on informal cultures in programming education. In more recent EUP and HCI research, the term 'tinkering' has been used to carry many of the implications of the French word [7] although without the useful theoretical precedents. All of these combine 
to suggest a concern with practical action and skill as an intrinsic motivation for EUP. We hypothesise that:

\section{(H1) People high on bricoleurism have intrinsic motivation for EUP if it enables them to manipulate and construct assemblages from components. This might be associated with ordering their online life, tinkering with things that might be useful, interest in how things work and pleasure in fixing things.}

\section{B. Technophilia}

There is a significant market in technology for its own sake - people interested in acquiring the latest products and skills as an exercise in fashion rather than utility $\lceil 10\rceil$. An interesting recent phenomenon has been the growing attention to 'coding' as an attainment that might be fashionable in itself (e.g. YearOfCode, CoderDojo, CodeClub etc [111). Technophilia is a variant of neophilia, as an intrinsic motivational factor for those who enjoy new experiences. Although this fashionable aspect to interest in coding is relatively recent, and hence not reported in the previous literature in EUP, we hypothesised that it might be observable among the current technology audience, and that it would be independent from the considerations of practical utility implicit in bricoleurism. We hypothesise that:

(H2) People with a high technophilia personality factor might have intrinsic motivation for EUP due to their curiosity about new technologies and learning how to use them. This might be associated with enjoyment of using new technologies and interest in innovative products.

\section{Artistry}

End-user programming has often been proposed as a route to enable more creative use of technology, dating back as far as Sutherland's Sketchpad, and Kay's Smalltalk. Although mainstream EUP research has tended to emphasise applications in business and home automation, there has recently been increasing interest in the use of digital technologies for creative artwork. This includes performance situations such as live coding [12], animated and interactive visual art such as that produced with the Processing language [13] or Palimpsest [14], and the embedded and mechatronic constructions of the maker movement, in which art projects incorporate software for platforms such as Arduino and Raspberry Pi [15]. We hypothesise that:

(H3) People with an artistry personality factor might have intrinsic motivation for EUP if it allows them to display creativity and originality. This might be associated with enjoyment in experimenting with creative ideas, as well as interest in sources of inspiration.

\section{EXPERIMENT 1: VALIDATING THE FACTORS}

This first experiment explores research question 1 , in order to validate the hypothetical intrinsic motivation factors. We used the MyPersonality dataset ${ }^{1}$, composed of over 6 million Facebook users, who completed the International Personality Item Pool (IPIP) FFM Personality Inventory [16]. Participants provided opt-in consent to use their scores and Facebook

http://mypersonality.org/ profile data in research. Their interests were determined from their Facebook "Likes".

\section{A. Method}

Following the standard approach in studies conducted with the MyPersonality dataset, we selected Facebook likes that a) occurred with moderately high frequency in the data set, and b) might be associated with one of the three hypothetical factors. For Bricoleurism, these were mainly home maintenance and DIY groups, for Technophilia, gadget magazines and technology news, and for Artistry, creative arts and media. We included 22 likes in each group (Table I).

TABLE I. FACEBOOK LIKES USED TO CLASSIFY THE THREE PREDICTED PERSONALITY TRAITS. THE WEBPAGE OF ANY PARTICULAR FACEBOOK LIKE CAN BE VIEWED AT HTTPS://WWW.FACEBOOK.COM/FACEBOOK_LIKE

\begin{tabular}{|l|l|}
\hline $\begin{array}{l}\text { Personality } \\
\text { Constructs }\end{array}$ & Facebook Likes \\
\hline Bricoleurism & $\begin{array}{l}\text { techcrunch, lifehacker, gizmodo, instructables, } \\
\text { diy_network, ehowcom, wikihow, snapon_tools, } \\
\text { woodworking, cooking, cooking_channel, } \\
\text { radioshack, computer_repair, gardening, } \\
\text { making_videos, better_recipes, buildcom, } \\
\text { office_depot, the_home_depot, flip_that_house }\end{array}$ \\
\hline $\begin{array}{l}\text { Game_Informer, computers, science, } \\
\text { video_games, ubuntu, linux, technology, } \\
\text { mathematics, engineering, computer_science, } \\
\text { html, programming, minecraft, star_trek, Intel, } \\
\text { web_design, i_robot, astronomy, } \\
\text { computer_programming, igncom, } \\
\text { web_development, Technology }\end{array}$ \\
$\begin{array}{l}\text { arts, music_player, music, painting, sculpting, } \\
\text { graphic_design, photography, the_artists_studio, } \\
\text { fine_arts, artist, musicians_institute, } \\
\text { the_art_institutes, drawing, artcom, ceramics, } \\
\text { beauty, sketching, design, designing, } \\
\text { taking_pictures }\end{array}$ \\
\hline Artistry
\end{tabular}

We next constructed a linear regression model, correlating these three sets of likes with the FFM personality factors as obtained from the MyPersonality dataset from participants who had recorded those likes in their profiles. We carried out a Principal Components Analysis (PCA) in order to determine whether the three sets of likes form disjoint clusters within this space. Finally, we calculated correlation for each of these clusters with the independent components of the FFM model, to provide an intrinsic motivation model for EUP that might distinguish between these hypothetical factors.

\section{B. Results}

Figure 1 shows PCA results from the three sets of Facebook likes. The three hypothetical alternative sets do form distinct clusters, with the Artistry and Technophilia clusters most clearly separated. The Bricoleurism cluster, as expected, has a degree of overlap with each. The centroids of the three clusters are clearly distinct. Inspection of the PCA coordinates confirms that there are plausible semantic associations between those likes that appear close together, and also that those in the overlap between the clusters share appropriate semantic associations (for example, web_design appears at the intersection of the Artistry and Technophilia clusters). 


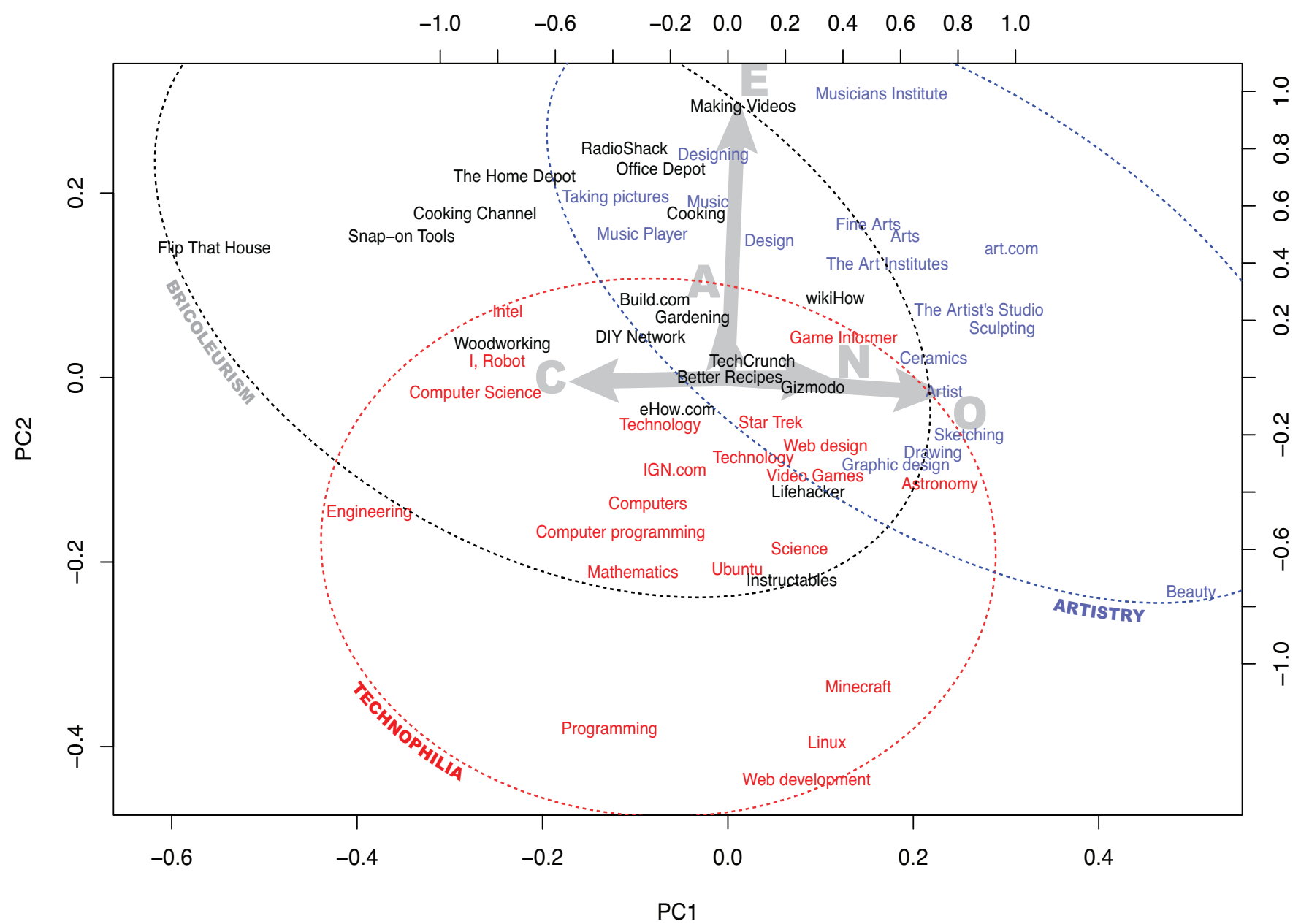

Fig. 1. Principal Components Analysis of the three sets of Facebook likes. Each set is grouped by colour and a containing ellipse labelled with the name of the set. The FFM personality components $(\mathrm{O}, \mathrm{C}, \mathrm{E}, \mathrm{A}, \mathrm{N})$ are shown as vectors mapped within the same PCA space. Various interesting properties can be observed, including the relatively large overlap of Bricoleurism and Technophilia, the inverse correlation of Technophilia with Extraversion, and the correlation of Artistry with Openness and Neuroticism.

Figure 1 also shows the FFM components O, C, E, A and N, as vectors mapped onto the same PCA dimensions. The visualisation confirms that the directions defined by these vectors in the PCA space are sufficiently orthogonal to distinguish between the centroids of the three clusters.

We separately calculated regression coefficients for each of the clustered sets of likes seen in Figure 1. The linear coefficients obtained from the parameters of the regression model are shown in Figure 2. As can be seen in the figure, the FFM traits Openness and Conscientiousness appear to discriminate between our hypothetical factors of Bricoleurism and Artistry, while Extraversion and Neuroticism discriminate between Artistry and Technophilia. Agreeableness is also negatively correlated with Technophilia. From visual inspection, it can be seen that each of the three hypothetical factors for intrinsic motivation in EUP is associated with a distinctive set of personality traits, including at least one inverse correlation distinguishing each of them. These results indicate that proposed hypothetical factors offer a useful account of the distinct personality traits that may underlie intrinsic motivation in EUP. In the next experiment, we test whether these factors are predictive of end-users' motivation.

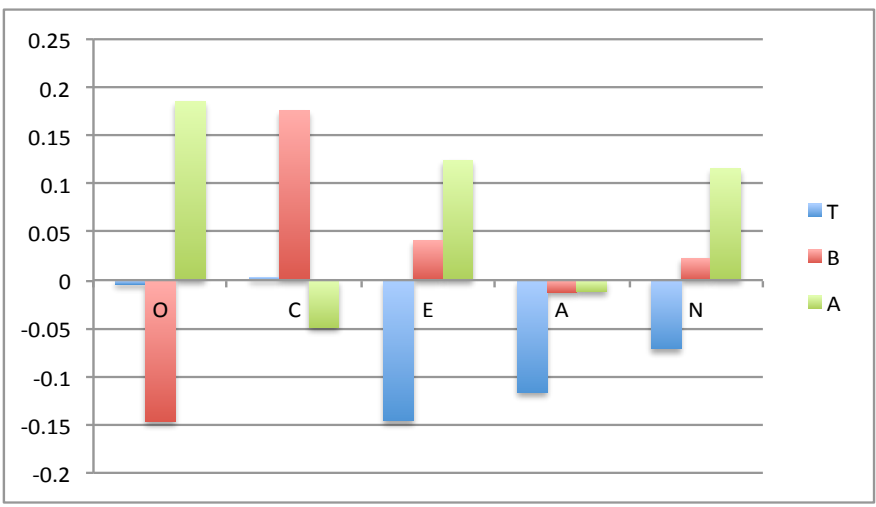

Fig. 2. Big-five personality traits profile projected for the three hypothesized personality types based on the MyPersonality dataset (T: Technophilia, B: Bricoleurism, A: Artistry, O: Openness, C: Conscientiousness, E: Extroversion, A: Agreeableness, N: Neuroticism) 


\section{EXPERIMENT 2A: APPLYING THE FACTORS}

Our second experiment explores research question 2, to test whether, for any given individual, it is possible to predict on the basis of their personality type what factors they are likely to find intrinsically motivating in EUP. The coefficients of the linear model developed in Experiment 1 can be used to define a hypothetical "motivation space" for end-user programming in terms of the three factors that we have introduced. The goal of this experiment is to test whether individuals' responses to EUP activity are consistent with their positions within that motivation space.

For each participant in this experiment, we use a standard personality questionnaire to identify a point describing that person within the space of intrinsic motivation factors, based on the coefficients of the linear model developed in Experiment 1 . We then compare this predicted combination of intrinsic motivation factors to the participant's own assessment of what they find satisfying in EUP.

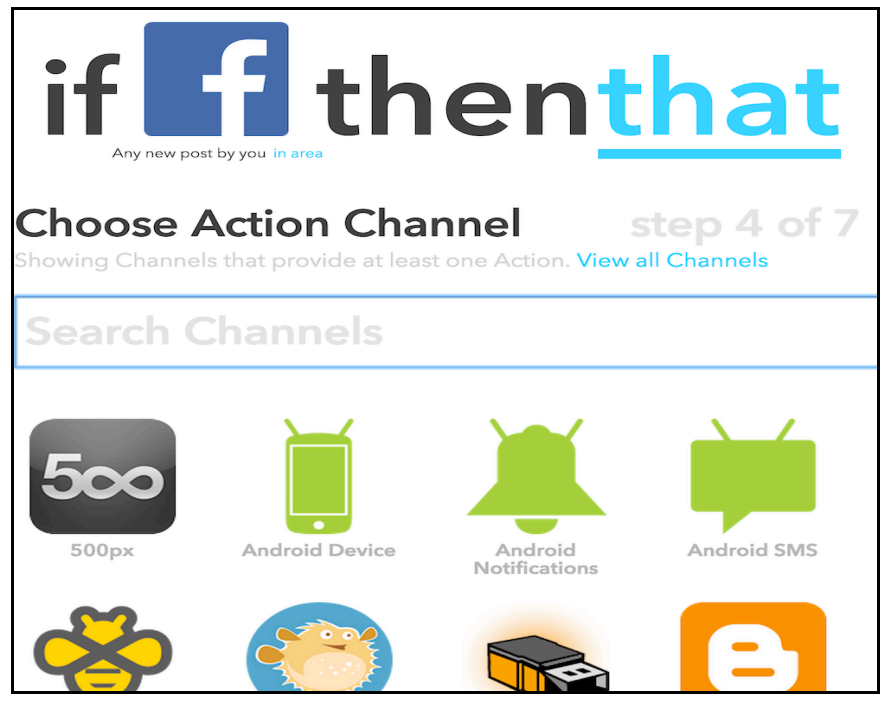

Fig. 3. Screenshot of IFTTT when creating a new recipe

The EUP environment used for this experiment is IFTTT (If This Then That), a popular online mashup authoring system that combines web services using simple condition-action rules that are described as "recipes" (Figure 3). Recipes created in IFTTT are persistent - they are assigned unique identifiers that can be browsed, collected and shared with other users.

\section{A. Participants}

We recruited 100 participants using the MicroWorkers ${ }^{2}$ crowd-sourcing platform. We recruited only from Englishspeaking countries. Each participant was paid 2.5 US dollars (\$2.50). Because the experiment involved completion of several long questionnaires, it was necessary to monitor the quality of responses, to exclude 'mental cheaters', who race through questionnaires with little thought or effort [17]. We therefore included 'speed trap' questions, such as "do not answer this question". We also checked for any unusual patterns in the participants' response such as giving the same answer for all (or nearly all) of the Likert scale questions. We discarded 10 of the 100 participants because of poor quality responses, leaving a total of 90 participants that have been used for the remaining analyses.

\section{B. Method}

The experiment was administered online, accessed via an entry page that presents the four phases of the experiment to be completed by the participant in order (Figure 4).

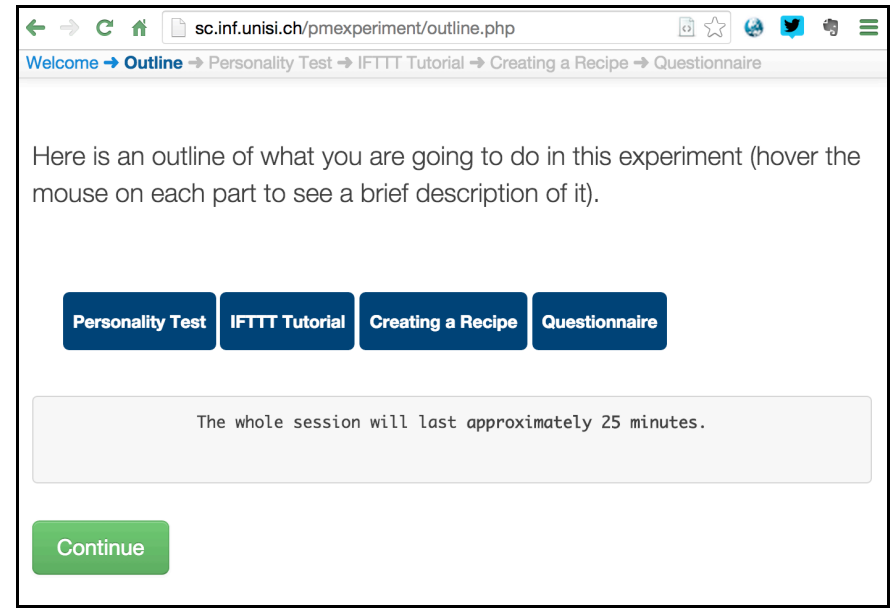

Fig. 4. Screenshot of entry page for online experiment

Participants first completed a standard personality questionnaire - the 50-item IPIP Big-Five factor markers. This is the same questionnaire used in the construction of the MyPersonality dataset. Participants were then asked to watch a short video tutorial explaining the operation of IFTTT. ${ }^{3}$ In the third and central part of the experiment, participants were asked to create a new user account on IFTTT, come up with an original idea for a new IFTTT recipe, and implement it using the IFTTT language. This task is intended to provide high external validity, being closely related (or even identical) to the sequence of actions that might be undertaken by a first-time user of IFTTT. After completing this task, they were asked to copy the link to the new recipe they had created, return to the experiment administration site, and paste the link into a form, so that we could inspect the recipe later.

After completing the EUP task, participants completed two questionnaires. The Intrinsic Motivation Inventory (IMI) [18] is a standard psychometric instrument that we used to assess the extent to which they had found the task itself intrinsically motivating. We also designed a questionnaire to evaluate the alternative aspects of intrinsic motivation explored in Experiment 1. This questionnaire (Figure 5) employs a controlled intrinsic motivation vocabulary - 'enjoy', 'interested', 'want' and 'like' - in relation to terms derived from our theoretical factors. The objective is to test whether, having completed an EUP task, participants describe their intrinsic motivation in ways that are consistent with predictions derived from the coefficients of the FFM model. 


\begin{tabular}{|c|c|c|c|c|}
\hline $\begin{array}{c}\text { Disagree } \\
\text { strongly } \\
\text { (1) }\end{array}$ & $\begin{array}{c}\text { Disagree } \\
\text { a little } \\
\text { (2) }\end{array}$ & $\begin{array}{c}\text { Neither agree } \\
\text { nor disagree } \\
\text { (3) }\end{array}$ & $\begin{array}{c}\text { Agree } \\
\text { a little } \\
\text { (4) }\end{array}$ & $\begin{array}{c}\text { Agree } \\
\text { strongly } \\
\text { (5) }\end{array}$ \\
\hline \multicolumn{5}{|c|}{$\begin{array}{l}\text { Bricoleurism } \\
\text { - I enjoy tinkering with things that might be useful } \\
\text { - I am interested in how things work } \\
\text { - I want to fix and build things } \\
\text { - I like to organise and put my digital life in order }\end{array}$} \\
\hline \multicolumn{5}{|c|}{$\begin{array}{l}\text { Technophilia } \\
\text { - I enjoy using new technologies } \\
\text { - I am interested in innovative products } \\
\text { - I want to try the latest app releases } \\
\text { - I like learning about high tech }\end{array}$} \\
\hline \multicolumn{5}{|c|}{$\begin{array}{l}\text { Artistry } \\
\text { - I enjoy experimenting with creative ideas } \\
\text { - I am interested in sources of inspirations } \\
\text { - I want to curate or collect sounds and images } \\
\text { - I like using attractive and aesthetic apps }\end{array}$} \\
\hline
\end{tabular}

Fig. 5. Questionnaire with 12 Likert scale questions to measure the degree of intrinsic motivational factors in end-user programming for the three predicted personality contructs.

\section{Results}

Using the results from Experiment 1, we created three coefficient functions as predictors of the three intrinsic motivation factors Bricoleurism (B), Technophilia (T), and Artistry (A). These functions are reported in the following equations used to derive a bscore (1), tscore (2) and ascore (3) respectively.

$$
\begin{aligned}
& b s c o r e=-0.146 o+0.176 c+0.041 e-0.012 a+0.022 n \\
& \text { tscore }=-0.004 o+0.003 c-0.145 e-0.116 a-0.071 n \\
& \text { ascore }=0.185 o-0.049 c+0.124 e-0.011 a+0.115 n
\end{aligned}
$$

Each of these functions is expressed in terms of normalized linear combinations (z-scores with mean $=0$, standard deviation=1) of the FFM personality traits $o, c, e, a$, and $n$ as seen in Figure 2 above. For each participant, we used the results from the IPIP questionnaire to calculate $o, c, e, a$, and $n$ values for that participant, and then used equations 1,2 and 3 to derive values for bscore, tscore, and ascore.

We also calculated reported intrinsic motivation for each factor using the Likert scale responses from the questionnaire in Figure 5. There are four questions related to each factor, and we used the mean of these four values to represent the participant's self-reported intrinsic motivation for each of the factors $\mathrm{B}, \mathrm{T}$ and $\mathrm{A}$.

We tested the hypothesis that it is possible to predictively distinguish between different individual intrinsic motivations for EUP on the basis of personality trait measures. For each of the three factors bscore, tscore and ascore we divided the data set into two halves, distinguishing between those participants having a low value for that score, and those having a high value. For each of these, the expected difference was observed in the self-reported intrinsic motivation, as shown in Figure 5 (independent t-test, $p<0.05$ ).

In order to verify that self-reported intrinsic motivation was indeed related to the overall degree of intrinsic motivation experienced by participants during the EUP task with IFTTT, we tested for correlation between the post-task Intrinsic Motivation Inventory (IMI) response, and each of the selfreported intrinsic motivation factors. We divided the participants to select those who were expected to be more motivated by each of the BAT factors. For those who were motivated by a particular factor, we would expect that their experience of being motivated by IFTTT would be positively correlated with that factor.

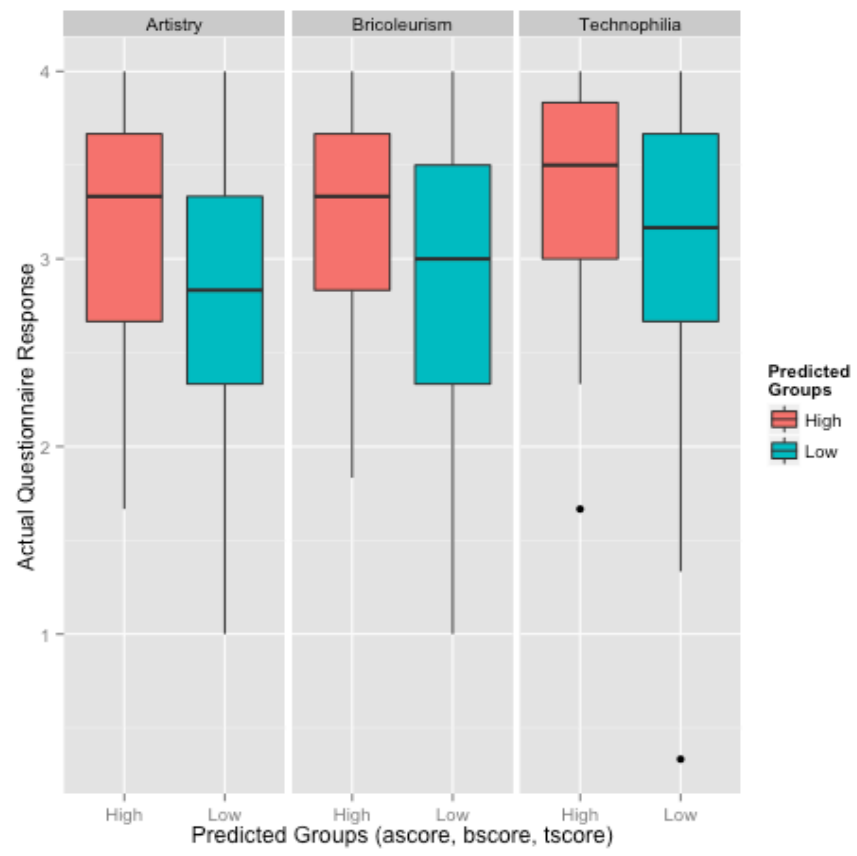

Fig. 6. A box plot showing the difference in the self-reported intrinsic motivation for each of the three intrinsic motivation factors (Artistry (A), Bricoleurism (B), and Technophilia (T)) when they are divided into two halves based on the motivation factor scores (i.e., ascore, bscore, and tscore).

We found that all three factors within the upper half of the participants (those participants who have a high value of bscore, tscore, and ascore respectively) were moderately positively correlated with IMI scores (Spearman's rank correlation rho $=0.6$, rho $=0.49$, rho $=0.54$ for, respectively, $\mathrm{B}, \mathrm{T}$, and $\mathrm{A}$ factors $-p<0.05$ ).

\section{EXPERIMENT 2B: REPLICATION OF 2A}

In order to test the robustness of the results from experiment $2 \mathrm{a}$ (section 5), we repeated it, employing the same method, but using a different Big-Five personality questionnaire as the basis for the $o, c, e, a, n$ values from which bscore, tscore, and ascore would be derived. In this replication, we therefore used the 44-item Big Five Inventory (BFI) [2] rather than the 50-item IPIP. 
We recruited 94 participants from the MicroWorkers website. As with the previous experiment, we filtered out poor quality responses, resulting in the discarding of 20 participants (leaving a total of 74 participants for the remaining analysis).

\section{A. Results}

As with the previous experiment, we observed a significant difference between the upper half and lower half of the sample for each of the BAT factors (independent t-test, $p<0.05$ ). As with the first experiment, we also found a moderate positive correlation between the IMI score and those participants who were more motivated by each of the three BAT factors (Spearman's rank correlation rho $=0.48$, rho $=0.64$, rho $=0.46$ for, respectively, $\mathrm{B}, \mathrm{T}$, and $\mathrm{A}$ factors, $p<0.05$ ).

This provides both a replication of the experimental result, and also confirms that our intrinsic motivation model is robust with respect to the generic Five-Factor Model of personality.

\section{DISCUSSION}

In this section, we discuss the potential value, scientific foundations, and applications that arise from this research.

\section{A. Value of Intrinsic Motivation Models}

There is increasing interest in the different kinds of user experience that might be inherent in programming tasks, by comparison to other varieties of user interface. Although it seems likely that different people have different kinds of experience, and indeed this is implicit in the distinction between "end-user programmers" and (implicitly normal) "programmers", there has been no previous attempt to construct a quantified psychometric model of the personality factors that might underlie these experiences.

These experiments have been designed to apply existing psychometric techniques - standardised questionnaires for personality type and intrinsic motivation - to study the factors that underlie individual differences in the experience of programming. In this initial study, our main concern has been to identify whether there is any evidence that consistent measurement of those differences is possible. We have therefore taken a relatively crude approach to characterising intrinsic motivation, drawing on previous literature to define factors distinguishing kinds of EUP experience that are as different as possible from each other. The goal at this stage is to identify whether there is any basis for further research to be done, rather than to claim that the three factors we have identified should be a definitive characterisation of intrinsic motivation in EUP.

\section{B. Five Factor Personality Model}

It should be noted that we treat the Five Factor personality model as defining a multidimensional space within which all members of the population are distributed. This paper is based on data collected from three different sample groups, each of which we assume to be drawn from the same overall population. The first of these is the very large research sample collected in the MyPersonality project, which we used to construct the correlation model that relates our three proposed intrinsic motivation factors to the FFM personality traits via their Facebook likes. The second and third samples are the experimental groups that we recruited via the MicroWorkers site, who completed two different standard FFM questionnaires as part of the experimental task.

We assume that these samples have been drawn from the same overall population, and that this population is homogeneous with respect to the five-factor personality model. This assumption of homogeneity is difficult to prove conclusively, and it is possible that there is a specific cultural bias distinguishing MicroWorkers participants from Facebook users. Nevertheless, it is plausible to assume that the two groups are similar in many respects (they are active users of the Internet, are likely to be drawn from younger demographics etc). Furthermore, given that we had to choose one personality model as a basis for broad population coverage, then the fivefactor model, with its associated standardised questionnaires, is the most extensively validated and normalised. Our replication using two different FFM questionnaires provides valuable evidence in support of this assumption.

\section{Applications}

If it is possible to predict reliably what aspects of EUP an individual is likely to find intrinsically motivating, then this suggests several application opportunities. In the context of programming education, the implicit motivation constructs of Bricoleurism, Technophilia and Artistry could either be predicted from prior psychometric assessments, or observed directly from other interests and activities of the student, in order to offer a range of teaching tools engaging with different types of motivation.

EUP products intended for use in the wider population could be evaluated in terms of these implicit motivation constructs, in order to predict where they might hold most appeal. Potentially, products could even configure themselves to emphasise different feature sets, different presentations of the user interface, specialist tutorials, or community support based on the expected motivations of different users. Once again, these might be predicted from psychometric measures (for example, access to an individual's Facebook profile can be used to estimate MyPersonality coordinates), or simply from observed usage of product features.

\section{Threats to Validity}

There are several cautions in interpreting this research, related to a) self-presentation and b) experimental demand. On social media platforms, people present themselves as having particular kinds of interest and motivation, but they may not be completely honest - for example, a person might say they 'Like' Minecraft on Facebook, but do so only to impress their friends rather than really liking it. Similarly, our MicroWorker participants may only have been pretending to be motivated, in order to guess our expectations and be rewarded for participating. This kind of issue is a constant concern in personality and motivation research. We have tried to apply current best practice from the field of psychometrics in social media, but others continuing this line of investigation in EUP research should be aware of these issues. 
An alternative approach would be to use our implicit motivation constructs in a direct measure of revealed preference, for example in the applications described in the previous section. The underlying personality measures offer a means of predicting and interpreting these in terms of the personality literature, but design applications need not rely on indirect assumptions in relation to personality.

\section{CONCLUSIONS}

We have reported two experiments as a first investigation of personality and intrinsic motivation factors in end-user programming. Our first research question was to explore what factors might cause EUP to be intrinsically motivating for different groups of individuals. We have identified three intrinsic motivation constructs, provisionally named Bricoleurism, Technophilia and Artistry, each of which describes a different aspect of intrinsic motivation in EUP. We have used the MyPersonality dataset to confirm that these three constructs are independent, and are correlated with different psychometric personality traits in a manner that means specific groups of individuals are likely to be motivated by different combinations of these factors.

Our second research question asked whether it is possible to measure, for any individual, the particular factors that will cause EUP to be intrinsically motivating for that person. We explored this through two experiments that were designed to maximize external validity, recruiting users online to use a currently popular EUP mashup tool. In addition to the EUP task, the participants in this experiment completed standardized psychometric personality questionnaires as well as a standardized assessment of intrinsic task motivation. We compared the predictions derived from our proposed intrinsic motivation constructs to participants' self-report of the ways in which they would be motivated to carry out EUP activities in future. The findings of this experiment suggest that there is future potential to measure and predict the aspects of EUP that will be intrinsically motivating for different users.

The findings of these experiments suggest that there is potential value in applying personality constructs to understand intrinsic motivation in EUP. The implicit motivation constructs that we have proposed, or others like them, have the potential to describe broad trends in the general population. They can be predicted, for any individual, in various ways - including prior personality measures, social media profiles, or revealed preferences in patterns of usage within an EUP product. As a result, we believe that access to EUP can be broadened, and that the behavior of EUP systems can be refined to better meet the needs of users.

\section{ACKNOWLEDGMENTS}

Alan Blackwell and Michal Kosinski's collaboration was funded by the Boeing Corporation. Saeed Aghaee's research was funded by the Swiss National Research Foundation. We are grateful to reviewers for helpful feedback on an earlier draft of this paper.

\section{REFERENCES}

$[1]$

M. Burnett, "The End-User Software Engineering Vision," in Opening presentation at the First Workshop on End-User Software Engineering (WEUSE I) In Conjunction with ICSE 2005, 2005.

[2] O. P. John and S. Srivastava, "The Big Five trait taxonomy: History, measurement, and theoretical perspectives," Handb. Personal. Theory Res., vol. 2, pp. 102-138, 1999.

P. T. Costa and R. R. McCrae, Professional manual: revised NEO personality inventory (NEO-PI-R) and NEO five-factor inventory (NEO-FFI). 1992

[4] S. Wray, "SQ minus EQ can predict programming aptitude," Proc. PPIG 19th Annu. Work. URL, pp. 243-254, 2007.

[5] B.-C. Catherine and D. D. Wheeler, "The Myers-Briggs Personality Type and Its Relationship to Computer Programming," J. Res. Comput. Educ., Feb. 2014.

[6] L. F. Capretz, "Personality types in software engineering," Int. J. Hum. Comput. Stud., vol. 58, no. 2, pp. 207-214, Feb. 2003.

[7] L. Beckwith, C. Kissinger, M. Burnett, S. Wiedenbeck, J. Lawrance, A. Blackwell, and C. Cook, "Tinkering and gender in end-user programmers' debugging," Proc. SIGCHI Conf. Hum. Factors Comput. Syst. - CHI '06, p. 231, 2006.

[8] A. F. Blackwell, J. A. Rode, and E. F. Toye, "How do we program the home? Gender, attention investment, and the psychology of programming at home," Int. J. Hum. Comput. Stud., vol. 67, pp. 324-341, 2009.

[9] B. S. Turkle and S. Papert, "Epistemological Pluralism and the Revaluation of the Concrete," Culture, vol. 11, pp. 1-32, 1992.

[10] G. Slade, Made to break: Technology and obsolescence in America. Harvard University Press, 2009.

[11] A. Mamolo, "Coding is Cool : TEACH Magazine." [Online]. Available: http://teachmag.com/archives/7626. [Accessed: 10-Feb2015].

[12] A. Blackwell, A. McLean, J. Noble, and J. Rohrhuber, "Collaboration and learning through live coding (Dagstuhl Seminar 13382)," Dagstuhl Reports, vol. 3, no. 9, pp. 130-168, 2014.

[13] C. Reas and B. Fry, Processing: A Programming Handbook for Visual Designers and Artists, vol. 54. 2007.

[14] A. F. Blackwell, "Palimpsest: A layered language for exploratory image processing," J. Vis. Lang. Comput., 2014.

[15] A. F. Blackwell, S. Aaron, and R. Drury, "Exploring Creative Learning for the Internet of Things era," in the Psychology of Programming Interest Group Annual Conference (PPIG 2014), 2014, pp. 147-158.

[16] L. R. Goldberg, "A broad-bandwidth, public domain, personality inventory measuring the lower-level facets of several five-factor models," Personal. Psychol. Eur., vol. 7, pp. 7-28, 1999.

[17] B. Albert, T. Tullis, and D. Tedesco, "Beyond the Usability Lab," Elsevier, vol. 1, p. 328, 2010.

[18] R. M. Ryan, "Control and information in the intrapersonal sphere: An extension of cognitive evaluation theory.," Journal of Personality and Social Psychology, vol. 43. pp. 450-461, 1982. 